\title{
Ontogenia de los esporangios y esporogénesis del helecho Phymatosorus scolopendria (Polypodiaceae)
}

\author{
Edgar Javier Rincón Barón ${ }^{1 *}$, Beatriz Elena Guerra ${ }^{1}$, Adriana Ximena Sandoval Meza ${ }^{1}$ \\ $\&$ Silvia Espinosa-Matías ${ }^{2}$
}

1. Grupo de Investigación Agroambiente y Salud-MICROBIOTA, Facultad de Ciencias de la Salud y Facultad de Ciencias Exactas Naturales y Agropecuarias, Universidad de Santander, calle 70 No 55-210, Campus Universitario Lagos del Cacique, Bucaramanga, Colombia; ed.rincon@mail.udes.edu.co, ejrbaron@gmail.com, bguerra@udes.edu.co, asandoval@udes.edu.co

2. Laboratorio de Microscopía Electrónica de Barrido, Facultad de Ciencias, Universidad Nacional Autónoma de México, Av. Universidad 3000, C.P. 04510, Ciudad de México, México; semfc2010@gmail.com

* Correspondencia

\section{Recibido 14-XI-2019. Corregido 23-III-2020. Aceptado 27-III-2020.}

\begin{abstract}
Ontogeny of sporangia and sporogenesis of the fern Phymatosorus scolopendria (Polypodiaceae). Introduction: Research about the ontogeny of sori, sporangia, receptacular paraphyses and sporogenesis of leptosporangiate ferns are scarce in the scientific literature. Objectives: To describe and analyze the ontogeny of sori, sporangia, receptacular paraphyses and sporogenesis of Phymatosorus scolopendria. Methods: Fertile fronds of $P$. scolopendria were collected in the campus of the Universidad de Antioquia, Medellín, Colombia, during the months March and May (annual rain season) of 2017. The fertile fronds of the samples at different developmental stages were fixed and processed according to the standard protocols for embedding and sectioning in paraffin and resin. Sections of $0.5 \mu \mathrm{m}$ obtained in resin were stained with Toluidine blue, which differentially stains primary and secondary walls, highlights the cell nucleus and sporopolenin and secondarily stains polyphenols. For detailed descriptions, additional sections were processed with Safranin-Alcian blue, allowing the distinction of components of primary and secondary walls, nuclei, cuticle and polyphenols; Hematoxylin-Alcian blue to enhance nuclei and primary walls and Phloroglucinol-HCl for lignin. Observations and photographic records were done with a photonic microscope. For the observations and descriptions with scanning electron microscopy (SEM), the sori were dehydrated with 2,2-dimethoxypropane, critical point dried and coated with gold. Results: The sori are exindusiate, superficial, vascularized and have mixed development; they are associated with uniseriate and multicellular receptacle paraphyses. During the development of the sori, the epidermal cells of the receptacle that will form the sporangia are the first differentiated followed by those forming the receptacle paraphyses. The sporangium is leptosporangiate, with long stalks formed by one or two cell rows. The annulus of the sporangia displays secondary walls with U-shaped thickenings rich in lignin. The meiosis is simultaneous and the spore tetrads are arranged in a decussate or tetragonal shape. The cellular tapetum is initially unistratified but becomes bistratified after a periclinal division. The cells of the internal strata of the cellular tapetum loose structural integrity giving rise to a plasmodial tapetum that invades the meiotic sporocytes. During the sporoderm development, the sporopollenin-composed exospore is the first formed followed by the endospore, composed by cellulose, pectin and carboxylated polysaccharides; the process ends with the perispore. Polyphenols were mainly detected on vacuoles in cells of the sporangium, paraphysis and receptacle. When the time comes for the spore maturation, the remnants of cellular and the plasmodial tapeta have fully degenerated. Abundant orbicles are seen near the spores in the sporangial cavity.
\end{abstract}

Rincón Barón, E.J., Guerra, B.E., Sandoval Meza, A.X., \& Espinosa-Matías, S. (2020). Ontogenia de los esporangios y esporogénesis del helecho Phymatosorus scolopendria (Polypodiaceae). Revista de Biología Tropical, 68(2), 655-668. 
Conclusions: The ontogeny of the sporangia and sporogenesis of $P$. scolopendria are similar to the previously described for leptosporangiate ferns. Furthermore, in $P$. scolopendria, the receptacle paraphyses of the sori have a role protecting the sporangium during the early development stages.

Key words: epiphytic fern, Microsoroideae, sporangia development, sorus, ultrastructure.

El género Phymatosorus Pic. Serm. es ampliamente reconocido y aceptado por la comunidad de pteridólogos (Hennipman, Veldhoen, \& Kramer, 1990; Bosman 1991; Smith, et al., 2006; Petchsri \& Boonkerd, 2014), este helecho pertenece a la familia Polypodiaceae y subfamilia Microsoroideae (Christenhusz, Zhang, \& Schneider, 2011). Sin embargo, Nooteboom (1997), lo considera como sinónimo de Microsorum Link., en este orden de ideas, la delimitación exacta de este último género es aún controversial y es considerado como polifilético, por lo que se requiere de un exhaustivo análisis sistemático que determine los límites del género (Kreier, Zhang, Muth, \& Schneider, 2008; PPG I, 2016; Testo, Field, Sessa, \& Sundue, 2019). En la presente investigación se siguen las propuestas de clasificación que reconocen a los dos géneros por separado (Bosman, 1991; Smith et al., 2006). Phymatosorus es un género con aproximadamente 12 especies ampliamente distribuidas en el paleotrópico; algunas de estas, se ha naturalizado exitosamente en el neotrópico (Hennipman et al., 1990; Tryon \& Lugardon, 1991; Tejero-Díez \& Torres, 2012; Petchsri \& Boonkerd, 2014). A pesar de que Phymatosorus scolopendria (Burm. f.) Pic. Serm. es una especie de valor ornamental, cultivada en viveros y jardines y que puede crecer espontáneamente sobre árboles y rocas, su distribución exacta en Colombia es incierta. Además, por ser una especie introducida, no se ha incluido hasta el momento en ninguna flora sobre helechos de este país.

A pesar de que los helechos leptosporangiados son diversos (PPG I, 2016; Sessa, 2018), son pocos los trabajos que describen de manera detallada los procesos de la ontogenia de los esporangios y la esporogénesis. En este sentido, cabe destacar los trabajos clásicos de Wilson (1958) y Pal y Pal (1963) quienes describen la ontogenia de los esporangios de Phlebodium aureum (L.) J. Sm. y Ceratopteris thalictroides (L.) Brongniart, respectivamente. Posteriormente, utilizando nuevas técnicas de preparación de muestras y observación, las descripciones sobre la ontogenia esporangial y la esporogénesis en helechos se han tornado más detalladas, al grado de incluir eventos subcelulares asociados a estos procesos (Peterson \& Kott, 1974; Bowen \& Williams, 1977; Sheffield \& Bell, 1979; Sheffield, Laird, \& Bell, 1983; Lugardon, 1990; Van Uffelen, 1990, 1992, 1993; Parkinson, 1995; Qiu, White, \& Turner, 1995; Churchill, Tryon, \& Barrington, 1998; González, Prada, \& Rolleri, 2010; Gabarayeva, Grigorjeva, \& Márquez, 2011; Triana-Moreno, 2012). En este sentido, Rincón, Guerra, Restrepo y Espinosa (2019) realizaron una descripción pormenorizada del desarrollo de los soros, ontogenia de los esporangios y escamas receptaculares, así como la esporogénesis del helecho Pleopeltis macrocarpa (Bory ex Willd.) Kaulf. En dicha investigación se aplicaron pruebas histoquímicas, cuyos resultados se relacionaron con aspectos fisiológicos relevantes, como, por ejemplo, la importancia de las escamas receptaculares en la protección e hidratación de los esporangios. Estos mismos autores han demostrado la importancia y conveniencia de utilizar técnicas modernas de microscopía y tinción para la descripción, con finos detalles, de los eventos reproductivos en helechos que permitan trascender de lo meramente descriptivo, a una aproximación morfofisiológica de estos eventos.

La presente investigación aporta información detallada sobre el desarrollo de los soros, ontogenia de los esporangios, paráfisis receptaculares y esporogénesis del helecho 
P. scolopendria. Se detallan aspectos estructurales y ultraestructuales de estos procesos; así como la importancia de la lignina presente en los anillos de los esporangios y de la esporopolenina en la pared de las esporas maduras. Finalmente, se analiza la ornamentación de las esporas y los procesos de formación y depósito del esporodermo.

\section{MATERIALES Y MÉTODOS}

Entre marzo y mayo 2017 (época lluviosa del año), se recolectaron más de 30 ejemplares fértiles de $P$. scolopendria que crecían en canastas ornamentales y en el suelo del campus de la Universidad de Antioquia en la ciudad de Medellín (6² $15^{\prime} 56^{\prime \prime} \mathrm{N}-75^{\circ} 34^{\prime} 09^{\prime}$ W). El material de referencia se depositó en el Herbario del Instituto de Biología de la Universidad de Antioquia (HUA) con código de recolección (Rincón 022).

Para los estudios del desarrollo de los soros y ontogenia de los esporangios se tomaron 30 frondas fértiles con estructuras reproductivas en diferentes momentos de maduración. Estas se fijaron en glutaraldehído al $2.5 \%$ en buffer fosfato, $\mathrm{pH} 7.2$ y $0.2 \mathrm{M}$ durante 24-48 horas a $6{ }^{\circ} \mathrm{C}$. Luego de la fijación, las muestras se lavaron en el mismo buffer y posteriormente con agua destilada para luego post-fijarlos con tetróxido de osmio al $2 \%$ por 4 horas a $6{ }^{\circ} \mathrm{C}$ en oscuridad y agitación constante. Después se deshidrataron durante una hora en cada concentración de la serie gradual de etanol, y durante 12 horas en etanol al $100 \%$ a $6{ }^{\circ} \mathrm{C}$. $\mathrm{Al}$ término de la deshidratación, se embebieron en mezclas progresivas de óxido de propileo-resina Spurr, por una semana a temperatura ambiente. Se hicieron varios cambios de resina pura durante seis días en constante agitación. La resina fue polimerizada a $60{ }^{\circ} \mathrm{C}$ por 48 horas. Se obtuvieron secciones de $0.4-0.5 \mu \mathrm{m}$ de grosor con cuchillas de vidrio en ultramicrótomo Leica ${ }^{\circledR}$, que se tiñeron con azul de Toluidina (TBO) en bórax al $1 \%$, pH 3.6 por 30-60 s. Muestras adicionales se fijaron en una mezcla de formol, etanol y ácido acético (FAA) por 24-48 horas a $6{ }^{\circ} \mathrm{C}$, se cortaron en fragmentos de $1 \mathrm{~cm}$ de longitud, se deshidrataron en la serie gradual de alcoholes y xilol (Ruzin, 1999) y se incluyeron en Paraplast plus (Mc Cormick ${ }^{\circledR}$ ) durante 12 horas a $55{ }^{\circ} \mathrm{C}$. Se obtuvieron secciones transversales con micrótomo rotatorio LEICA RM $2125^{\circledR}$, entre 5-7 $\mu \mathrm{m}$ de grosor. Finalmente, se procedió a la tinción de las muestras como se indica en la Tabla 1 (Ruzin, 1999; Soukup 2014; Yeung, Stasolla, Sumner \& Huang 2015; Demarco 2017).

TABLA 1

Tinciones aplicadas a las secciones de los soros, esporangios y esporas de $P$. scolopendria

TABLE 1

Stains applied to the sections to sori, sporangia and spores of $P$. scolopendria

\section{TINCIÓN}

Azul de Toluidina (TBO) alciano (Fasga)

Hematoxilina de Gill IIIAzul de alciano

Técnica de Wiesner (Fluoroglucinol-HCL)

\section{COMPUESTO/ESTRUCTURA QUE RESALTA}

- Paredes secundarias lignificadas (xilema y esclerénquima), se tiñen de color verde o azul verdoso.

- Paredes primarias con polisacáridos carboxilados y ácido péctico (parénquima, colénquima, floema, cambium), se tiñen de color púrpura o púrpura rojizo.

- Polifenoles se tiñen de azul grisáceo.

- Esporopolenina se tiñe de azul turquesa.

- Paredes primarias se tiñen de azul.

- Paredes secundarias, cutícula, núcleos, suberina y polifenoles se tiñen de color rojo o fucsia.

- Tiñe paredes celulares primarias en azul, núcleos en color violeta oscuro, polifenoles marrón oscuro y lignina marrón claro.

- Específico para lignina (grupos coniferil aldehído y sinapil aldehído), se tiñe en color rojo.

- La tinción no es permanente.

- No se observan reacciones cruzadas con otros compuestos o estructuras. 


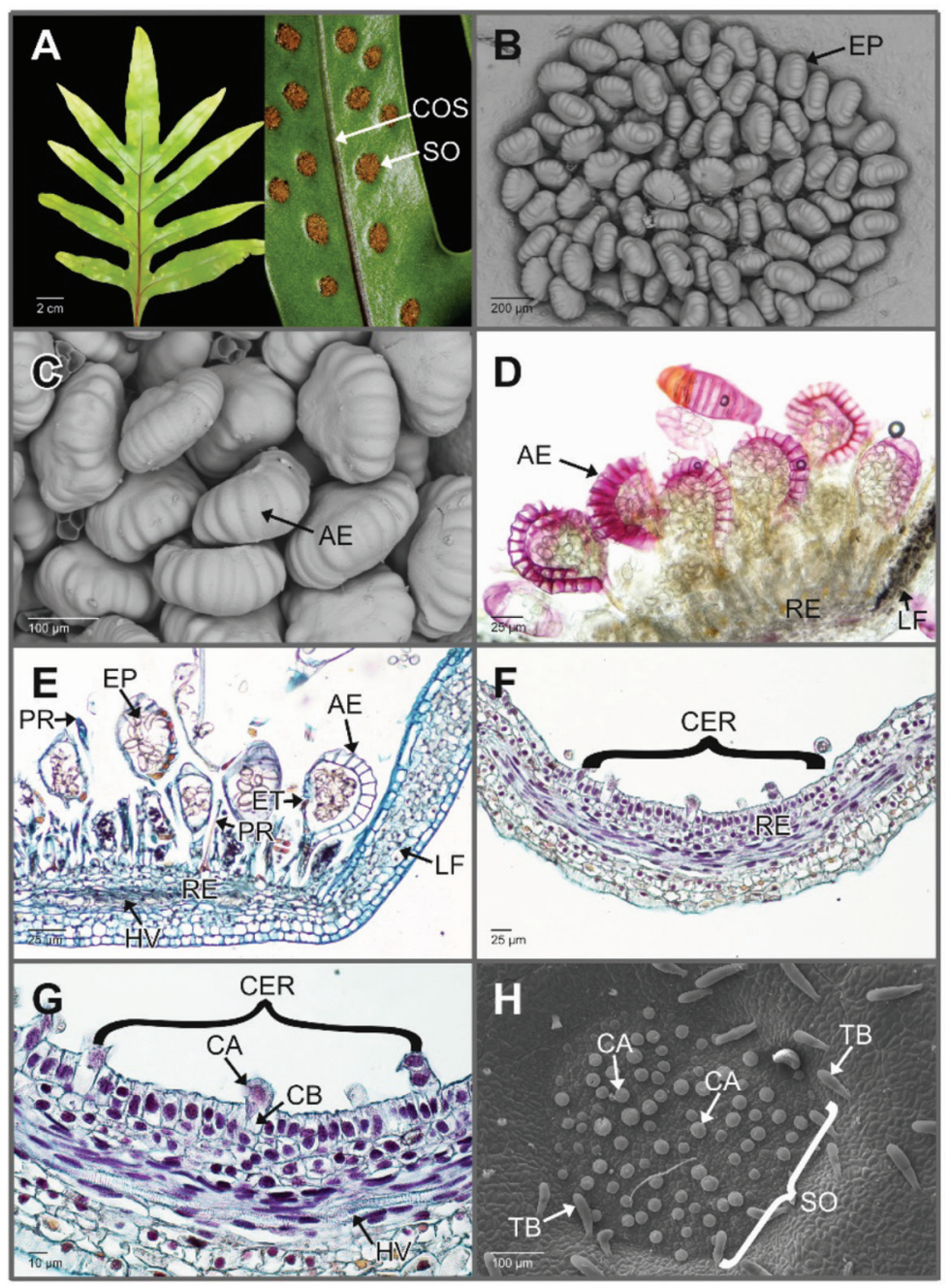

Fig. 1. Phymatosorus scolopendria. Soros, esporangios maduros y células receptaculares A. Frondas fértiles, mostrando los pares de lóbulos y la distribución de los soros hundidos. B-C. Detalle de los soros exindusiados y leptosporangios con anillos engrosados; en ambos casos, las paráfisis no se aprecian (Microscopía electrónica de barrido-MEB). D. Sección longitudinal a mano alzada de un soro maduro. Observe la lignificación del anillo del esporangio en color púrpura (Floroglucinol ácido). E. Sección longitudinal de un soro maduro. Se observan esporangios en diferentes etapas del desarrollo y la relación estrecha del soro con los haces vasculares (Hematoxilina-azul de alciano). F-G. Zona del receptáculo mostrando las características citológicas de las células epidérmicas (Hematoxilina-azul de alciano). H. Soro inmaduro, se muestran primordios de esporangios y tricomas bicelulares (MEB). AE: anillo del esporangio; CA: célula apical; CB: célula basal; CER: células epidérmicas del receptáculo; COS: cóstula; EP: esporangios; ET: estomio; LF: lamina foliar; HV: haces vasculares; PR: paráfisis receptaculares; RE: receptáculo; SO: soro; TB: tricomas bicelulares.

Fig. 1. Phymatosorus scolopendria. Sori, mature sporangia and cells of the receptacle. A. Fertile fronds showing pairs of lobes and the distribution of sunken sori. B-C. Detail of the exindusiate sori and leptosporangia with thickened annulus (Scanning electron microscopy-SEM). D. Freehand cross section of a mature sorus. The highly lignified annulus of the sporangium is visible in purple staining (Phloroglucinol-HCl). E. Cross section of a mature sorus. The sporangia on different development stages are shown, as well as the close relation between the sorus and the vascular bundles (HematoxylinAlcian blue). F-G. Receptacle zone showing the cytological features of the epidermal cells. H. An immature sorus where two-celled trichomes and sporangial primordia are visible (SEM). AE: sporangial annulus; CA: apical cell; CB: basal cell; CER: epidermal cells of the receptacle; COS: costule; EP: sporangia; ET: stomium; LF: leaf blade; HV: vascular bundles; PR: receptacle paraphyses; RE: receptacle; SO: sori; TB: two celled trichomes. 
Para llevar a cabo las descripciones morfológicas con MEB, fragmentos de las frondas con soros se fijaron en Glutaraldehído como se indicó anteriormente y se deshidrataron en 2,2 dimetoxipropano (Lin, Falk, \& Stocking, 1977) para posteriormente desecar a punto crítico con un desecador SAMDRI ${ }^{\circledR}-795$. Se montaron sobre cinta conductiva de carbono de doble cara y se recubrieron con oro en una ionizadora DENTON VACUUM DESK IV durante 5 minutos. Las observaciones y registro fotográfico se realizaron en un microscopio electrónico de barrido JEOL JSM-6490LV.

Las secciones en resina y parafina se examinaron con un microscopio fotónico Nikon $80 \mathrm{i}$ eclipse ${ }^{\circledR}$ y Nikon eclipse-Ni con el sistema de contraste diferencial de interferencia (CDI). Las fotografías se obtuvieron con cámara digital Nikon DS-Fi1 ${ }^{\circledR}$ utilizando el programa NIS Elements versión 4.30.02 de Nikon. Los términos botánicos empleados para las descripciones se usaron sensu Lellinger (2002) y Punt, Hoen, Blackmore, Nilsson y Le Thomas (2007). El término banda de orgánulos se utiliza según Brown y Lemmon (2001a), el de cámaras plasmodiales según Rincón, Forero, Gélvez, Torres y Rolleri (2011) y orbículas, según Tryon y Lugardon (1991) y Passarelli, Gabriel, Prada y Rolleri (2010).

\section{RESULTADOS}

Las frondas fértiles de $P$. scolopendria son simples, profundamente pinnatífidas y con amplias alas a lo largo del raquis, el número de pares de lóbulos por fronda fue de cinco o menos (Fig. 1A). Las frondas por lo general son de color verde amarillento o verde brillante. Los soros son exindusiados redondeados o ligeramente oblongos, se encuentran hundidos en un receptáculo profundo; por lo general, formando una o dos filas irregulares a cada lado de la cóstula. La venación es poco conspicua e inmersa en la lámina (Fig. 1A). P. scolopendria tiene leptosporangios de pedicelos largos y con dos filas de células, anillo en posición vertical con 14-16 células lignificadas; además, se observa un evidente estomio (Fig. 1B, Fig. 1C,
Fig. 1D, Fig. 1E). Los soros son superficiales y asociados al tejido vascular; su desarrollo es mixto, es decir, en un mismo soro hay esporangios en diferentes etapas del desarrollo (Fig. 1D, Fig. 1E). Los soros también se caracterizan por tener paráfisis multicelulares uniseriadas, las cuales en la madurez son inconspicuas y difíciles de observar debido a que los esporangios dificultan su visualización (Fig. 1E).

El soro inicia su desarrollo en el receptáculo, como una depresión en la cara abaxial de la lámina foliar (Fig. 1F). En sección longitudinal, las células epidérmicas del receptáculo son alargadas con núcleos prominentes y paredes primarias delgadas (Fig. 1G). Estas células, por divisiones mitóticas, darán origen en primer lugar a los esporangios y posteriormente a las paráfisis. Las células que formaran a los esporangios se dividen por un plano oblicuo dando origen a la célula basal y a la célula apical que posteriormente se diferenciarán en el primordio del esporangio (Fig. 1G, Fig. 1H). En la cara abaxial de la lámina foliar se observan tricomas bicelulares constituidos por una célula basal corta y otra distal alargada de ápice redondeado. Estos tricomas se distribuyen por toda la lámina hasta el límite con el receptáculo del soro (Fig. 1H).

Una vez que se han diferenciado la célula basal y apical, éstas experimentan sucesivas divisiones mitóticas en varios planos para formar una estructura alargada que corresponde al pedicelo y una porción expandida terminal globosa que corresponde a la incipiente cápsula del esporangio (Fig. 2A, Fig. 2B). Para esta etapa del desarrollo, se observan los primordios de las paráfisis.

A medida que avanza el desarrollo de los esporangios, los esporocitos premeióticos se rodean de un grupo de células parietales estériles a partir de las cuales por divisiones mitóticas anticlinales y periclinales se formará la cápsula del esporangio. Posterior a este evento, numerosas células epidérmicas del receptáculo, experimentan divisiones mitóticas sucesivas en un plano periclinal-transversal hasta formar estructuras multicelulares uniseriadas, que corresponden a las paráfisis, de 


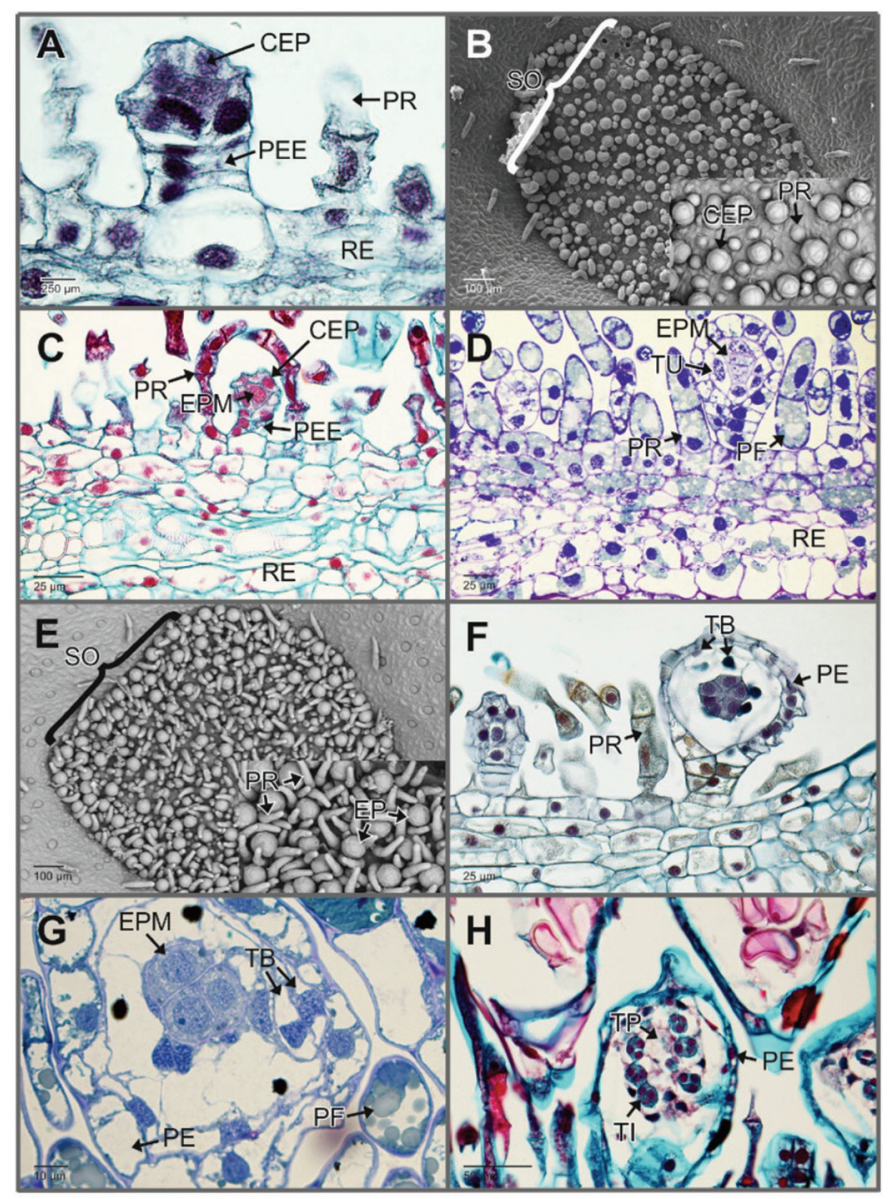

Fig. 2. Phymatosorus scolopendria. Ontogenia de los esporangios y paráfisis receptaculares. A. Esporangios y paráfisis receptaculares indiferenciadas (Hematoxilina-azul de alciano). B. Acercamiento al soro inmaduro; en el recuadro se observan los primordios de los esporangios y las paráfisis (Microscopía electrónica de barrido-MEB). C. Los pedicelos, las cápsulas de los esporangios y paráfisis han experimentado divisiones mitóticas. Los esporocitos premeióticos se han diferenciado y se aprecian rodeados por una capa de células parietales; las paráfisis se observan pluricelulares (Safranina-azul de alciano-FASGA). D. Sección longitudinal del soro, el tapete celular uniestratificado se ha diferenciado. Note los depósitos de polifenoles (Toluidina). E. Detalle morfológico de D., en el recuadro las paráfisis maduras se curvan hacia los esporangios (MEB). F-G. Diferenciación del tapete celular biestratificado (Hematoxilinaazul de alciano y Toluidina, respectivamente). H. Tétrada de esporas con tapete plasmodial persistente (FASGA). CEP: cápsula del esporangio; EPM: esporocitos premeióticos; EP: esporangios; PE: pared de los esporangios; PEE: pedicelos de los esporangios; PF: polifenoles; PR: paráfisis receptaculares; RE: receptáculo; SO: soro; TB: tapete celular biestratificado TI: tétrada inmadura; TP: tapete plasmodial; TU: tapete celular uniestratificado.

Fig. 2. Phymatosorus scolopendria. Ontogeny of the sporangia and receptacle paraphyses. A. Sporangia and receptacle paraphyses are undifferentiated (Hematoxylin-Alcian blue). B. Close up of the immature sorus, the box shows the sporangia and paraphyses primordia (Scanning electron microscopy-SEM). C. The stalks, capsules of the sporangia and paraphyses became divided by mitosis. The premeiotic sporocytes are differentiated, now surrounded by a layer of parietal cells; paraphyses are pluricellular (Safranine-Alcian blue-FASGA). D. Longitudinal section of the sorus, the unistratified cellular tapetum become differentiated. Notice the deposits of polyphenolic nature (Toluidine). E. Morphology detail of D., the box shows the mature paraphyses curving towards the sporangium (SEM). F-G. Differentiation of the bistratified cellular tapetum (Hematoxylin-Alcian blue and Toluidine, respectively). H. Spore tetrad and persistent plasmodial tapetum (FASGA). CEP: capsule of the sporangium; EPM: premeiotic sporocytes; EP: sporangia; PE: sporangial wall; PEE: stalks of the sporangia; PF: polyphenols; PR: receptacle paraphyses; RE: receptacle; SO: sorus; TB: bistratified cellular tapetum; TI: immature tetrad; TP: plasmodial tapetum; TU: unistratified cellular tapetum. 
apariencia tricomatosa, que persistirán hasta la maduración de los esporangios y liberación de las esporas (Fig. 2C, Fig. 2D).

Los esporocitos premeióticos se dividen mitóticamente en plano periclinal para formar la capa de células tapetales iniciales (Fig. 2C, Fig. 2D). En esta etapa, las células del receptáculo, paráfisis y esporangios mostraron vacuolas con depósitos de polifenoles (Fig. 2D). Para este preciso momento del desarrollo, las paráfisis uniseriadas han madurado y se han curvado hacia los esporangios protegiéndolos (Fig. 2E). Posteriormente, las células tapetales iniciales se dividen periclinalmente y forman un tapete celular biestratificado, que delimita a los esporocitos premeióticos (Fig. 2F, Fig. 2G). Estos esporocitos, se caracterizan por presentar escaso citoplasma de aspecto granular, un núcleo central voluminoso, uno o dos nucléolos y cromosomas condensados; los polifenoles se aprecian aún en esta etapa del desarrollo del soro (Fig. 2G). Los esporocitos premeióticos se dividen mitóticamente para aumentar su número. Simultáneamente, el estrato interno del tapete celular pierde su integridad estructural y se transforma en un tapete plasmodial que invade la cavidad esporangial y separa individualmente a los esporocitos, los cuales, una vez aislados, experimentan meiosis (Fig. 2H, Fig. 3A).

El tapete plasmodial persiste hasta la maduración de las esporas (Fig. 3B, Fig. 3C, Fig. 3D). La meiosis es de tipo simultánea y por consiguiente las esporas solo se separan hasta el final de dicho proceso (Fig. 3B, Fig. $3 \mathrm{C}$ ). No se observó la banda de orgánulos (Fig. 3A, Fig. 3B, Fig. 3C). Para el momento de la formación de las tétradas, el tapete plasmodial las rodea formando cámaras plasmodiales (Fig. 3B, Fig. 3C). El tapete plasmodial se caracteriza por presentar núcleos voluminosos, sin nucléolos evidentes y la masa citoplasmática se aprecia granular (Fig. 3B, Fig. 3C). Al final de la meiosis, las esporas en la tétrada se disponen de forma decusada o tetragonal (Fig. 3A, Fig. 3B, Fig. 3C).

Una vez que la meiosis ha concluido, las esporas jóvenes se separan de la tétrada en cámaras plasmodiales individuales, en las cuales se diferencian y alcanzan la madurez y posterior liberación; en estas cámaras se observaron orbículas de esporopolenina (Fig. 3D). Para el momento de liberación de las esporas, el tapete plasmodial y el estrato externo del tapete celular han degenerado por completo y en la cavidad del esporangio se aprecia abundantes orbículas (Fig. 3E). En el esporangio, el anillo se ha diferenciado por completo y presenta engrosamientos sectorizados lignificados en forma de "U" (Fig. 3D, Fig. 3F).

En el proceso de diferenciación del esporodermo, primero se deposita el exosporio, seguido del endosporio y finalmente el perisporio (Fig. 3B, Fig. 3C, Fig. 3D, Fig. 3E). El exosporio es la capa más gruesa del esporodermo y está formado por esporopolenina, teñido en color azul turquesa (Toluidina), le sigue en grosor el endosporio formado de materiales de pared primaria, que se ven de color violeta con la tinción de Toluidina (Fig. 3E). El perisporio es la capa más delgada, también conformada de materiales de pared primaria, posiblemente de celulosa y pectinas. (Fig. 3E). Las esporas maduras son monoletes con lesura engrosada, de ornamentación rugada con pequeñas orbículas esféricas distribuidas azarosamente sobre el esporodermo (Fig. 3F, Fig. 3G, Fig. 3H).

\section{DISCUSIÓN}

Las observaciones de la presente investigación concuerdan con lo presentado por Petchsri y Boonkerd (2014), quienes estudiaron especímenes de Microsorum y Phymatosorus de Tailandia, y determinaron que este último género se caracteriza porque los soros son exindusiados y se encuentran en receptáculos profundamente hundidos formando una a dos filas discontinuas al lado y lado de la cóstula y las frondas son profundamente pinnatífidas o algunas veces pinnaticompuestas. Por el contrario, Microsorum presenta soros superficiales y frondas simples a pinanatifidas. De igual forma se coincide con Petchsri y Boonkerd (2014) y Possley y Howell (2015) quienes indican que $P$. scolopendria se diferencia de 


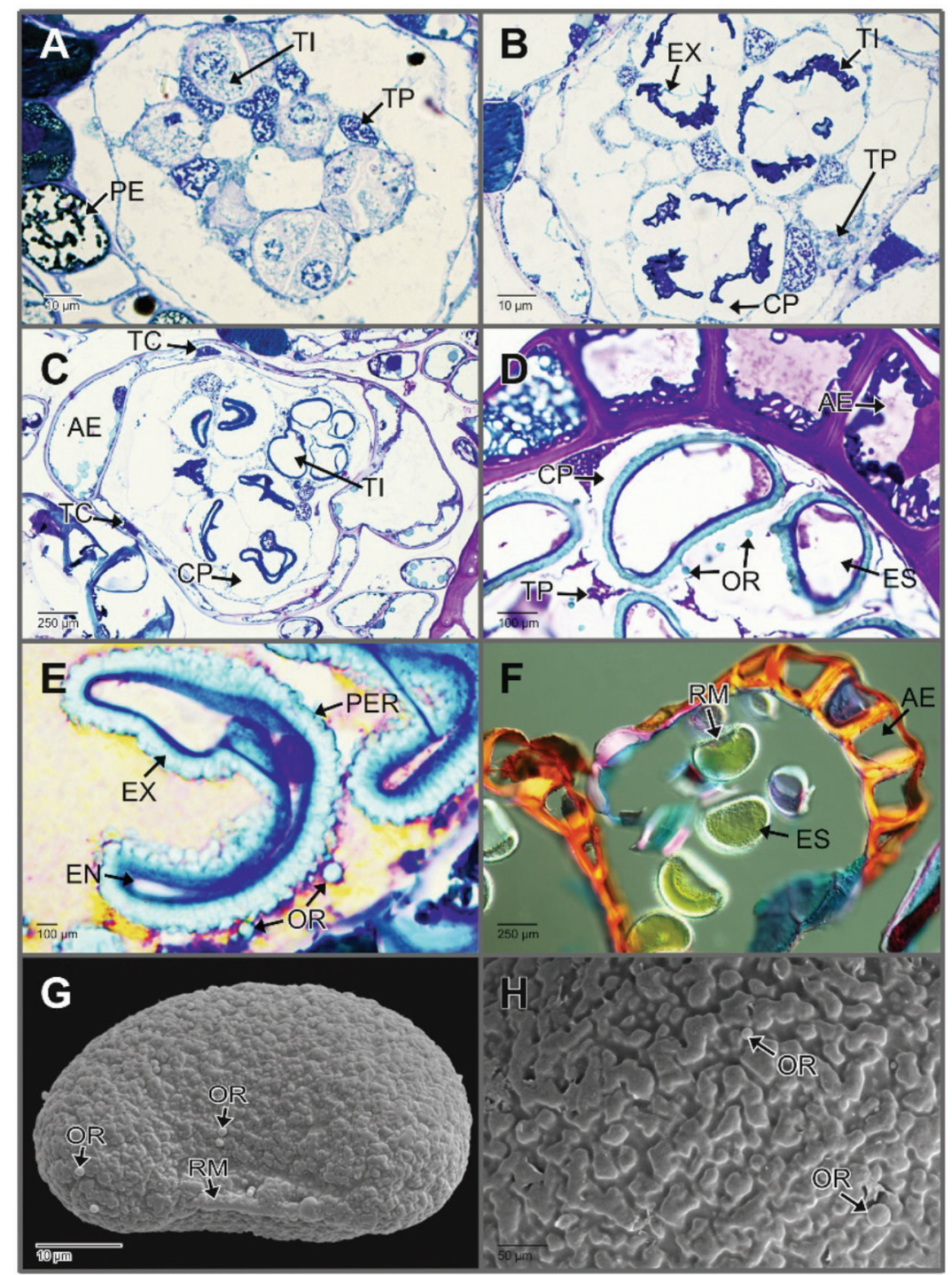

Fig. 3. Phymatosorus scolopendria. Tétradas de esporas, anillo del esporangio y esporas maduras. A. Tétradas inmaduras rodeadas por el tapete plasmodial(Toluidina). B-F. Desarrollo de las esporas y diferenciación del anillo del esporangio (Toluidina A-E. y Safranina-azul de alciano-FASGA F.). B-C. Tétradas de esporas en disposición decusada o tetragonal. El estrato externo del tapete celular persiste. D. Esporas en cámaras plasmodiales individuales. E. Detalle del perisporio, exosporio y endosporio. G-H. Esporas monoletes maduras con ornamentación rugada y orbículas (Microscopía electrónica de barrido-MEB). AE: anillo del esporangio; CP: cámaras plasmodiales; ES: esporas; EN: endosporio; EX: exosporio; OR: orbículas; PE: pared del esporangio; PER: perisporio; RM: lesura monolete; TC: estrato externo del tapete celular; TI: tétrada inmadura; TP: tapete plasmodial.

Fig. 3. Phymatosorus scolopendria. Spore tetrads, sporangial annulus and mature spores. A. Immature tetrads surrounded by the plasmodial tapetum (Toluidine). B-F. Development of the spores and differentiation of the sporangial annulus (Toluidine A-E. and Safranine-Alcian blue-FASGA F.). B-C. Spore tetrads tetragonal or decussate shaped. The external strata of the cellular tapetum is preserved. D. Spores on individual plasmodial gaps. E. Details of the perispore, exospore and endospore. G-H. Mature monolete spores with rugate sculpture and orbicles (Scanning electron microscopy-SEM). AE: sporangial annulus; CP: plasmodial gaps; ES: spores; EN: endospore; EX: exospore; OR: orbicles; PE: sporangial wall; PER: perispore; RM: monolete leasure; TC: external strata of the cellular tapetum; TI: immature tetrad; TP: plasmodial tapetum. 
las demás especies del género por presentar cinco o menos pares de lóbulos por fronda; la venación de los lóbulos es inconspicua, debido a que estas se encuentran inmersas en los tejidos de la lámina foliar. Adicionalmente, se observó que $P$. scolopendria presenta paráfisis receptaculares multicelulares uniseriadas y esporas amarillentas, elipsoidales, monoletes y rugadas; que es coincidente con lo registrado previamente para esta especie (Hennipman et al., 1990; Tryon \& Lugardon, 1991; Petchsri \& Boonkerd, 2014).

Aun cuando el género Phymatosorus (sensu Bosman, 1991) es reconocido en varias investigaciones (Hennipman, et al., 1990; Smith et al., 2006; Petchsri \& Boonkerd, 2014). En otros trabajos, es considerado dentro del género Microsorum; entre otras razones, debido a que pueden formar híbridos intergenéricos (Bostock \& Spokes, 1998). Además, Nooteboom (1997) indica que se pueden presentar interpretaciones erróneas cuando se aplican los términos catádromo y anádromo en relación a la venación areolada tal y como lo presenta Bosman (1991) en su trabajo monográfico sobre helechos Microsoroideae y en el cual reconoce al género Phymatosorus. Sin embargo, es importante tener en cuenta que, a pesar de los esfuerzos hechos hasta el momento sobre la taxonomía y la filogenia de estos grupos de plantas, la circunscripción genérica dentro de la familia Microsoroideae es aún controversial (Kreier et al., 2008; PPGI, 2016, Testo et al., 2019). Así mismo, se ha demostrado ampliamente que el género Microsorum no es monofilético (Kreier et al., 2008; Testo \& Sundue, 2016; Nitta, Amer \& Davis, 2018; Testo et al., 2019) lo cual sugiere una reconsideración del género.

En P. scolopendria los soros son de desarrollo mixto, en ellos se observaron esporangios en diferentes etapas del desarrollo, lo cual es congruente con observaciones previas para helechos leptosporangiados (Bower, 1963; Gifford \& Foster, 1989, Triana-Moreno, 2012; Rincón et al., 2019). Así mismo, se demostró que los soros de P. scolopendria son vascularizados, carácter que comparte con $P$. macrocarpa (Rincón et al., 2019), pero discrepa con las observaciones hechas por Schölch (2003) quien indica que en Polypodiacea los soros son carentes de vascularización. Esta controversia sugiere ampliar las investigaciones anatómicas a otras especies de esta familia para determinar la variabilidad en ausencia/presencia de tejido vascular y sus implicaciones.

La presencia de soros con desarrollo mixto podría ser una estrategia importante para la producción de esporas en diferentes momentos ecológicos, permitiendo la supervivencia de los gametófitos (Rincón et al., 2019). No obstante, esta hipótesis requiere de estudios ecológicos y reproductivos que permitan entender el valor adaptativo de este atributo (Gifford \& Foster, 1989; Qiu et al., 1995; Kumar, 2001).

Las características morfocitológicas de las células epidérmicas del receptáculo de $P$. scolopendria son similares a las descritas para otras especies de helechos (Wilson, 1958; Gifford, \& Foster, 1989; Qiu et al., 1995; Kumar, 2001; González et al., 2010; Triana-Moreno, 2012; Rincón et al., 2019). Sin embargo, se pueden presentar variaciones morfológicas entre los taxones, como lo observó Parkinson (1995) en las descripciones de la esporogénesis en Schizaea pectinata (L.) Sw. Este autor las describe como células de contorno globoso y protuberantes que se diferencian de las células adyacentes. A pesar de estas diferencias morfológicas, los procesos posteriores de división mitótica que experimentan estas células son similares a lo observado en helechos leptosporangiados.

De acuerdo con Peterson y Kott (1974) y Rincón et al., (2019) y la presente investigación, las etapas iniciales del desarrollo de los esporangios y las paráfisis receptaculares son similares en términos de división celular y posteriormente divergen para diferenciarse en estas estructuras. Es importante indicar que, aunque los esporangios y paráfisis receptaculares comparten el mismo origen; estos claramente presentan vías diferenciadas del desarrollo que determinan la formación de estructuras estériles de protección y estructuras fértiles capaces de generar esporas por meiosis. 
En P. scolopendria y otras especies, se ha demostrado que las divisiones que experimentan las células receptaculares para la diferenciación de los primordios de los esporangios, las características citológicas de los esporocitos premeióticos, así como las divisiones que estos experimentan para diferenciarse en el tapete celular, son similares y al parecer son procesos conservados en helechos leptosporangiados (Wilson, 1958; Peterson \& Kott, 1974; Bowen \& Williams, 1977; Sheffield \& Bell, 1979; Qiu et al., 1995; Passarelli et al., 2010; Rincón et al., 2019).

En los helechos leptosporangiados, por lo general, se forma un tapete plasmodial. En etapas tempranas de la ontogenia del esporangio, este es inicialmente celular uniestratificado y posteriormente se torna biestratificado. Finalmente, la capa más interna del tapete pierde la integridad celular y da origen a un plasmodio que rodea a los esporocitos, mientras que la capa externa persiste hasta la liberación de las esporas (Wilson, 1958; Peterson \& Kott, 1974; Sheffield \& Bell, 1979; Lugardon, 1990; Parkinson, 1995; Parkinson \& Pacini, 1995; Rincón et al., 2019). Estas observaciones son similares a lo observado en $P$. scolopendria. Así mismo, se ha demostrado que el tapete se relaciona con la producción de sustancias de reserva y estructurales para la síntesis de precursores de la pared de las esporas (Lugardon, 1990; Pacini \& Franchi, 1993; González et al., 2010; Rincón et al., 2019). En P. scolopendria se observó la formación de orbículas de esporopolenina principalmente al concluir la meiosis y una vez que las esporas se han separado en cámaras plasmodiales individuales, se determinó que las orbículas persisten hasta la madurez de las esporas. Estas observaciones soportan la idea de la función secretora de precursores de pared por parte del tapete, en esta especie en estudio. Adicionalmente, es importante indicar, que estas características también se han registrado para otras especies de helechos y licófitos (Uehara \& Kurita 1991; Rincón, Torres, \& Rolleri, 2013; Rincón, Rolleri, Alzate, \& Dorado, 2014a; Rincón, Rolleri, Passarelli, Espinosa, \& Torres 2014b). Por otro lado, el origen y función de las cámaras plasmodiales que rodean a los esporocitos en $P$. scolopendria, son similares a lo observado para especies de Psilotaceae y para helechos leptosporangiados (Parkinson, 1987; Lehmann, Neidhart \& Schlenkermann, 1984; Uehara \& Kurita, 1989; Rincón et al., 2013; Rincón et al., 2019).

En P. scolopendria la meiosis es simultánea y se relaciona con la formación de tétradas en posición decusada o tetragonal y la formación de esporas monoletes. Lo cual es congruente con lo observado por Rincón et al., (2019) para P. macrocarpa, pero difiere a lo observado por Furness, Rudall y Sampson (2002) quienes indicaron que este tipo de división se relaciona con la formación de tétradas de esporas en disposición tetraedral, y que por lo general se caracterizan por formar esporas triletes. Es importante indicar que, aunque Furness et al., (2002) hacen una exhaustiva investigación sobre estos procesos, estas se enfocan principalmente en angiospermas; por lo que ampliar los estudios a otros grupos de plantas, es recomendable.

La banda de orgánulos cumple una función importante indicando el lugar exacto del depósito de la placa celular y por consiguiente en la forma como se organizaran las futuras esporas jóvenes para conformar a la tétrada (Sheffield \& Bell, 1979; Sheffield et al., 1983; Brown \& Lemmon, 2001a; Brown \& Lemmon, 2001b; Rincón et al., 2013; Rincón et al., 2019) y aunque en esta investigación no se pudo observar, tal vez por la falta de detalle de algunas secciones, es probablemente que la función en $P$. scolopendria sea similar a la descrita para otras especies de helechos y licófitos.

La síntesis del esporodermo en licófitos y helechos homosporados es un proceso en el cual primero se forma el exosporio, posteriormente, el endosporio y al final se deposita el perisporio (Lugardgon, 1990; Tryon \& Lugardon, 1991; Uehara \& Kurita, 1991; Rincón et al., 2014a; Rincón et al., 2014b; Rincón et al., 2019). Esta secuencia de desarrollo es congruente a lo descrito en esta investigación para P. scolopendria. Así mismo, son similares a los registrados para helechos y licófitos heterospóricos, aunque 
con algunas diferencias en estructura (Tryon \& Lugardon, 1991; Uehara, Kurita, Sahashi, \& Ohmoto, 1991; Morbelli, 1995; Wellman, 2004). Debido a la similitud en la síntesis del esporodermo entre los diferentes grupos de helechos y licófitos se infiere que es un proceso conservado en este grupo de plantas.

En la presente investigación y empleando técnicas microscópicas selectas se confirmó el patrón de ornamentación rugada y la presencia de orbículas asociadas al esporodermo y en la cavidad del esporangio de $P$. scolopendria y son coincidentes con observaciones previas para esta especie (Tryon \& Tryon, 1982; Tryon \& Lugardon, 1991).

Así como en P. scolopendria, en otros trabajos se ha documentado la presencia de polifenoles en las células de los esporangios, paráfisis y células receptaculares (Peterson \& Kott, 1974; Rincón et al., 2019). La presencia de estos metabolitos secundarios podría estar relacionada con la respuesta de la planta para evitar la herbivoría y protección contra la radiación ultravioleta, como se ha descrito ampliamente en diferentes grupos de plantas (Lattanzio, Lattanzio, \& Cardinali, 2006; Kulbat, 2016).

\section{AGRADECIMIENTOS}

Los autores agradecen a las siguientes instituciones y personas: al laboratorio de Investigación e Innovación en Biotecnología Agroambiental (LIIBAAM) de la Universidad de Santander (UDES), al Laboratorio de Biotecnología y Centro de Microscopía Avanzada, Sede de Investigación Universitaria -SIU- de la Universidad de Antioquia (UdeA) por su apoyo en la observación de algunas de las muestras en microscopía fotónica y SEM respectivamente. A Marcelo Arana de la Universidad Nacional de Río Cuarto (Argentina) y Weston Testo de la Universidad de la Florida (USA) por su comunicación personal en relación de la taxonomía del grupo. También a Roberto Enrique LlanosRomero del Laboratorio de Química. Departamento de Ecología y Recursos Naturales.
Facultad de Ciencias, (UNAM) por su ayuda con las secciones bilingües del manuscrito

\section{RESUMEN}

Introducción: Las investigaciones sobre ontogenia de los soros, esporangios, paráfisis receptaculares y esporogénesis de los helechos leptosporangiados son escasas en la literatura científica. Objectivos: Describir y analizar la ontogenia de los soros, esporangios, paráfisis receptaculares y esporogénesis de Phymatosorus scolopendria. Métodos: Entre marzo y mayo 2017 (época lluviosa del año) se recolectaron frondas fértiles de $P$. scolopendria en el campus de la Universidad de Antioquia, Medellín-Colombia. Las frondas fértiles, en diferentes etapas del desarrollo se fijaron y procesaron de acuerdo a protocolos estándar para la inclusión y corte en parafina y resina. Las secciones de $0.5 \mu \mathrm{m}$ obtenidas en resina se tiñeron con azul de Toluidina que tiñe diferencialmente paredes primarias y secundarias, resalta núcleos celulares, y esporopolenina y de manera secundaria tiñe polifenoles. Para descripciones detalladas, otros cortes se tiñeron con Safranina-azul de alciano que discrimina entre componentes de pared primaria, secundaria, núcleos, cutícula y polifenoles; Hematoxilina-azul de alciano para resaltar núcleos y paredes primarias y Fluoroglucinol ácido para detectar lignina. Las observaciones y registro fotográfico se efectuaron con microscopio fotónico. Para la observación y descripción con microscopía electrónica de barrido (MEB), los soros se deshidrataron con 2,2 dimetoxipropano, se desecaron a punto crítico y se metalizaron con oro. Resultados: Los soros son exindusiados, superficiales, vascularizados y de desarrollo mixto, se encuentran asociados a paráfisis receptaculares multicelulares uniseriadas. Durante el desarrollo del soro primero se diferencian las células epidérmicas receptaculares que darán origen a los esporangios y posteriormente las células que originarán a las paráfísis receptaculares. El esporangio es de tipo leptosporangio de pedicelos largos de una o dos filas de células. Los anillos de los esporangios muestran paredes secundarias con engrosamientos en forma de "U" ricos en lignina. La meiosis es simultánea y las tétradas de esporas se disponen de forma decusada o tetragonal. El tapete celular es inicialmente uniestratificado pero por una división mitótica de tipo periclinal, se torna biestratificado. Las células del estrato interno del tapete pierden la integridad estructural dando origen a un tapete plasmodial que invade los esporocitos en meiosis, el estrato externo persiste hasta la etapa de esporas maduras. En las diferentes etapas de desarrollo del esporodermo, primero se forma el exosporio, compuesto por esporopolenina, seguida del endosporio, conformado por celulosa, pectina y polisacáridos carboxilados y finalmente el perisporio. Los polifenoles fueron detectados, principalmente, en las vacuolas de las células de los esporangios, paráfisis y células receptaculares. Para el momento de la liberación de las esporas, tanto la capa externa del tapete celular como 
el plasmodial han degenerado por completo. En la cavidad esporangial se aprecian orbículas adyacentes a las esporas. Conclusiones: la ontogenia de los esporangios y esporogénesis de $P$. scolopendria es similar al descrito previamente para helechos leptosporangiados. Adicionalmente, se indica que las paráfisis receptaculares presentes en los soros de $P$. scolopendria tienen la función de protección de los esporangios durante las primeras etapas del desarrollo.

Palabras clave: desarrollo de los esporangios, helecho epífito, Microsoroideae, soro, ultraestructura.

\section{REFERENCIAS}

Bosman, M.T.M. (1991). A monograph of the fern genus Microsorum (Polypodiaceae) including an attempt towards a reconstruction of the phylogenetic history of the microsoroids. Leiden Botanical Series, 14(1), $1-161$.

Bostock, P.D., \& Spokes, T.M. (1998). Polypodiaceae. In P.M. McCarthy (ed.), Flora of Australia (Vol. 48, pp. 66-84). Melbourne, Australia: BRS and CSIRO.

Bowen, W., \& Williams, D. (1977). Development of sporangia in Polypodium aureum var. undulatum: Initial scanning electron microscopical observations. Arkansas Academy of Science Proceedings, 31, 26-28.

Bower, F.O. (1963). The ferns (Filicales). Vols. I-III Reprint edn. New Delhi, India: Today and Tomorrow's Book Agency.

Brown, R.C., \& Lemmon, B.E. (2001a). Sporogenesis in eusporangiate ferns: I. Monoplastidic meiosis in Angiopteris (Marattiales). Journal of Plant Research, 114(3), 223-23.

Brown, R.C., \& Lemmon, B.E. (2001b). Sporogenesis in eusporangiate ferns: II. Polyplastidic meiosis in Ophioglossum (Ophioglossales). Journal of Plant Research, 114(3), 237-246.

Churchill, H., Tryon, R., \& Barrington, D.S. (1998). Development of the sorus in tree ferns: Dicksoniaceae. Canadian Journal of Botany, 76, 1245-1252.

Christenhusz, M., Zhang, X.C., \& Schneider, H. (2011). A linear sequence of extant families and genera of lycophytes and ferns. Phytotaxa, 19, 7-54.

Demarco, D. (2017). Histochemical analysis of plant secretory structures. En C. Pellicciari \& M. Biggiogera (Eds.), Histochemistry of single molecules methods and protocols (pp. 313-330). New York, U.S.A.: Humana Press.

Furness, C.A., Rudall, J.P., \& Sampson, F.B. (2002). Evolution of microsporogenesis in angiosperms. International Journal of Plant Science, 163(2), 35-260.
Gabarayeva, N.I., Grigorjeva, V.V., \& Márquez, G. (2011). Ultrastructure and development during meiosis and the tetrad period of sporogenesis in the leptosporangiate fern Alsophila setosa (Cyatheaceae) compared with corresponding stages in Psilotum nudum (Psilotaceae). Grana, 50, 235-261.

Gifford, M.E., \& Foster, S.A. (1989). Morphology and evolution of vascular plants. New York, U.S.A.: W. H. Freeman and Company.

González, G.E., Prada, C., \& Rolleri, C.H. (2010). Nuevo recuento cromosómico para Blechnum hastatum (Blechnaceae-Pteridophyta), con un estudio de la ontogenia y tipos de leptoporangios adultos. Gayana Botanica, 67(1), 52-64.

Hennipman, E., Veldhoen, P., \& Kramer, K.U. (1990). Polypodiaceae. En K.U. Kramer \& P.S. Green (Eds.), The Families and Genera of Vascular Plants. I Pteridophytes and Gymnosperms (pp. 203-230). Berlin, Germany: Springer-Verlag.

Kreier, H.P., Zhang, X.C., Muth, H., \& Schneider, H. (2008). The microsoroid ferns: Inferring the relationships of a highly diverse lineage of paleotropical epiphytic ferns (Polypodiaceae, Polypodiopsida). Molecular Phylogenetics and Evolution, 48(3), 1155-1167.

Kulbat, K. (2016). The role of phenolic compounds in plant resistance. Biotechnology and Food Science, 80(2), 97-108.

Kumar, K. (2001). Reproductive Biology of Pteridophytes. En B.M. Johri \& P.S. Srivastava (Eds.), Reproductive Biology of Plants (pp. 175-214). New York, U.S.A.: Springer-Verlag.

Lattanzio, V., Lattanzio, M.T.V., \& Cardinali, A. (2006). Role of phenolics in the resistance mechanisms of plants against fungal pathogens and insects. En F. Imperato (Ed.), Phytochemistry: advances in research (pp. 23-67). Trivandrum, India: Research Signpost.

Lellinger, D.B. (2002). A modern multilingual glossary for taxonomic pteridology. Pteridologia, 3, 1-263.

Lehmann, H., Neidhart, K.M., \& Schlenkermann, G. (1984). Ultrastructural investigations on sporogenesis in Equisetum fluviatile. Protoplasma, 123(1), 38-47.

Lin, C.H., Falk, R.H., \& Stocking, C.R. (1977). Rapid chemical dehydration of plant material for light and electron microscopy with 2,2-dimethoxypropane and 2,2-diethoxypropane. American Journal of Botany, 64(5), 602-605.

Lugardon, B. (1990). Pteridophyte sporogenesis: a survey of spore wall ontogeny and fine structure in a polyphyletic plant group. En S. Blackmore \& R.B. Knox (Eds.), Microspores: evolution and ontogeny (pp. 95-120). London, England: Academic Press. 
Morbelli, M.A. (1995). Megaspore wall in Lycophyta ultrastructure and function. Review of Palaeobotany and Palynology, 85, 1-12.

Nitta, J.H., Amer, S., \& Davis, C.C. (2018). Microsorum $\times$ tohieaense (Polypodiaceae), a new hybrid fern from French Polynesia, with implications for the taxonomy of Microsorum. Systematic Botany, 43(2), 397-413.

Nooteboom, H.P. (1997). The microsoroid ferns (Polypodiaceae). Blumea, 42(2), 261-395.

Pacini, E. \& Franchi, G.G. (1993). Role of the tapetum in pollen and spore dispersal. Plant Systematics and Evolution, 7, 1-11

Pal, N., \& Pal, S. (1963). Studies on morphology and affinity of the Parkeriaceae. II. Sporo-genesis, development of the gametophyte, and cytology of Ceratopteris thalictroides. Botanical Gazette, 124, 405-412.

Parkinson, B.M. (1987). Tapetal organization during sporogenesis in Psilotum nudum. Annals of Botany, 60, $353-360$.

Parkinson, B.M. (1995). Development of the sporangia and associated structures in Schizaea pectinata (Schizaeaceae: Pteridophyta). Canadian Journal Botany, $73,1867-1877$

Parkinson, B.M., \& Pacini, E. (1995). A comparison of tapetal structure and function in pteridophytes and angiosperms. Plant Systematics and Evolution, 198, $55-88$.

Passarelli, L.M., Gabriel, J.G., Prada, C., \& Rolleri, C.H. (2010). Spore morphology and ornamentation in the genus Blechnum (Blechnaceae). Grana, 49(4), 243-262.

Peterson, R.L., \& Kott, L.S. (1974). The sorus of Polypodium virginianum: some aspects of the development and structure of paraphyses and sporangia. Canadian Journal of Botany, 52, 2283-2288.

Petchsri, S., \& Boonkerd, T. (2014). The genera Microsorum and Phymatosorus (Polypodiaceae) in Thailand. Tropical Natural History, 14(2), 45-74.

PPG I. (2016). A community-derived classification for extant lycophytes and ferns. Journal of Systematics and Evolution, 54, 563-603.

Possley, J., \& Howell, P.L. (2015). Misidentification of "Microsorum scolopendria" in South Florida. American Fern Journal, 105(2), 127-130.

Punt, W., Hoen, P.P., Blackmore, S., Nilsson, S., \& Le Thomas, A. (2007). Glossary of pollen and spore terminology. Review of Palaeobotany and Palynology, 143(1-2), 1-83.
Qiu, Y.J., White, R.A., \& Turner, M.D. (1995). The developmental anatomy of Metaxya rostrata (Filicales: Metaxyaceae). American Journal of Botany, 82(8), 969-981.

Rincón, B.E.J., Forero, B.H.G., Gélvez, L.L.V., Torres, G.A., \& Rolleri, C.H. (2011). Ontogenia de los estróbilos, desarrollo de los esporangios y esporogénesis de Equisetum giganteum (Equisetaceae) en los Andes de Colombia. Revista de Biología Tropical, 59(4), $1845-1858$

Rincón, B.E.J., Torres, G.A., \& Rolleri, C.H. (2013). Esporogénesis y esporas de Equisetum bogotense (Equisetaceae) de las áreas montañosas de Colombia. Revista de Biología Tropical, 61(3), 1067-1081.

Rincón, B.E.J., Rolleri, C.H., Alzate, G.F., \& Dorado, G.J.M. (2014a). Ontogenia de los esporangios, formación y citoquímica de esporas en licopodios (Lycopodiaceae) colombianos. Revista de Biología Tropical, 62(1), 273-298.

Rincón, B.E.J., Rolleri, C.H., Passarelli, M.L., Espinosa, M.S., \& Torres, G.A.M. (2014b). Esporogénesis, esporodermo y ornamentación de esporas maduras en Lycopodiaceae. Revista de Biología Tropical, 62(3), 1161-1195.

Rincón, B.E.J., Guerra, S.B.H., Restrepo, Z.D.E. \& Espinosa, M.S. (2019). Ontogenia e histoquímica de los esporangios y escamas receptaculares del helecho epífito Pleopeltis macrocarpa (Polypodiaceae). Revista de Biología Tropical, 67(6), 1292-1312.

Ruzin, S.E. (1999). Plant microtechnique and microscopy. New York, U.S.A.: Oxford University.

Schölch., A. (2003). Relations between submarginal and marginal sori in ferns III. Superficial sori with emphasis on Pteridaceae and morphological relations to marginal sori. Plant Systematics and Evolution, 240, 21-233.

Sessa, B.E. (2018). Evolution and classification of ferns and Lycophytes. En H. Fernández (Ed.), Current advances in fern research (pp. 179-200). Cham, Switzerland: Springer.

Sheffield, E., \& Bell, P.R. (1979). Ultrastructural aspects of sporogenesis in a fern, Pteridium aquilinum (L.) Kuhn. Annals of Botany, 44, 393-405.

Sheffield, E., Laird, S., \& Bell, P.R. (1983). Ultrastructural aspects of sporogenesis in the apogamous fern Dryopteris borreri. Journal of Cell Science, 63, 125-134.

Smith, A.R., Pryer, K.M., Schuettpelz, E., Korall, P., Schneider, H., \& Wolf, P.G. (2006). A classification for extant ferns. Taxon, 55(3), 705-731.

Soukup, A. (2014). Selected simple methods of plant cell wall histochemistry and staining for light microscopy. 
En V. Žárský, \& F. Cvrčková (Eds.), Plant cell morphogenesis: methods and protocols, methods in molecular biology (pp. 25-40). New York, U.S.A.: Humana Press.

Tejero-Díez, J.D., \& Torres-Díaz, A.N. (2012). Phymatosorus grossus (Polypodiaceae) en México y comentarios sobre otros pteridobiontes no-nativos. Acta Botánica Mexicana, 98, 111-124.

Testo, W.L., Field, A.R., Sessa, E.B., \& Sundue, M. (2019). Phylogenetic and morphological analyses support the resurrection of Dendroconche and the recognition of two new genera in Polypodiaceae subfamily Microsoroideae. Systematic Botany, 44(4), 1-16.

Testo, W. \& Sundue, M. (2016). A 4000-species dataset provides new insight into the evolution of ferns. Molecular Phylogenetics and Evolution, 105, 200-211.

Triana-Moreno, L.A. (2012). Desarrollo del esporangio en Pecluma eurybasis var. villosa (Polypodiaceae). Boletín Cientifico. Centro de Museos. Museo de Historia Natural, 16(2), 60-66.

Tryon, R.M., \& Tryon, A.F. (1982). Ferns and allied plants, with special reference to tropical America. New York, U.S.A.: Springer.

Tryon, A.F., \& Lugardon, B. (1991). Spores of the Pteridophyta: surface, wall structure, and diversity based on electron microscope studies. Nueva York, U.S.A.: Springer.

Uehara, K., \& Kurita, S. (1989). An ultrastructural study of spore wall morphogenesis in Equisetum arvense. American Journal of Botany, 76(7), 939-951.
Uehara, K. \& Kurita, S. (1991). Ultrastructural study on spore wall morphogenesis in Lycopodium clavatum (Lycopodiaceae). American Journal Botany, 78(1), 24-36.

Uehara, K., Kurita, S., Sahashi, N., \& Ohmoto, T. (1991). Ultrastructural study of microspore wall morphogenesis in Isoëtes japonica (Isoëtaceae). American Journal of Botany, 78, 1182-1190.

Van Uffelen, G.A. (1990). Sporogenesis in Polypodiaceae (Filicales). I. Drynaria sparsisora (Desv.) Moore. Blumea, 35, 177-215.

Van Uffelen, G.A. (1992). Sporogenesis in Polypodiaceae (Filicales). II. The genera Microgramma Presl and Belvisia Mirbel. Blumea, 36, 515-540.

Van Uffelen, G.A. (1993). Sporogenesis in Polypodiaceae (Filicales). III. Species of several genera. Spore characters and their value in phylogenetic analysis. Blumea, 37, 529-561.

Wellman, C.H. (2004). Origin, function and development of the spore wall in early land plants. En A.R. Hemsley \& I. Pole (Eds.), The evolution of plant physiolo$g y$ (pp. 43-63). Linnean Society Symposium, Number 21. San Diego, U.S.A.: Academic Press.

Wilson, K.A. (1958). Ontogeny of the sporangium of Phlebodium (Polypodium) aureum. American Journal of Botany, 45(6), 483-491.

Yeung, E.C.T., Stasolla, C., Sumner, M.J., \& Huang, B.Q. (2015). Plant microtechniques and protocols. New York, U.S.A.: Springer. 